\title{
Hospitalisations pour une blastomycose dans le nord-ouest de l'Ontario, de 2006 à 2015
}

\author{
S Litvinjenko1*, D Lunny ${ }^{2}$
}

\section{Résumé}

Contexte : La blastomycose, causée par l'organisme Blastomyces dermatitidis, est une maladie fongique invasive présente dans le centre du Canada ainsi que dans le centre et le Midwest des États-Unis.

Objectif : Décrire les tendances et l'épidémiologie relatives aux cas de blastomycose hospitalisés parmi les résidents du nord-ouest de l'Ontario, de 2006 à 2015.

\begin{abstract}
Méthodologie : Les données concernant les hospitalisations pour une blastomycose ont été extraites de la base de données sur les congés des patients (BDCP), accessible par l'intermédiaire de Savoir-Santé Ontario. La BDCP comprend des renseignements administratifs, cliniques et démographiques sur les congés fournis par l'Institut canadien d'information sur la santé (ICIS). Les dossiers sur la blastomycose ont été répertoriés à l'aide des codes de la classification internationale des maladies 10 (CIM-10) B40.0 à B40.9. Les taux d'hospitalisation ont été calculés pour l'ensemble de l'Ontario, et les taux d'hospitalisation selon l'âge ont été calculés pour le nord-ouest de l'Ontario et analysés selon la région sanitaire, le moment et la saisonnalité ainsi que les symptômes initiaux.
\end{abstract}

Résultats : Au cours de cette période de 10 ans, on a signalé 581 hospitalisations pour une blastomycose en Ontario, dont 245 (42\%) dans le nord-ouest de la province, bien que cette région ne compte que pour $0,6 \%$ de la population. Le taux moyen d'hospitalisation pour une blastomycose dans le nord-ouest de l'Ontario était de 35,0 pour 100000 personnes par année. Ce taux variait de 1,7 dans la région de Red Lake à 57,9 dans la région de Kenora. Le tableau clinique le plus courant était des symptômes pulmonaires aigus. Les hommes étaient 1,36 fois plus susceptibles d'être hospitalisés pour une blastomycose que les femmes (intervalle de confiance $[I C]$ à $95 \%$ de 1,06 à 1,$75 ; p<0,05)$. La plupart des hospitalisations ont été enregistrées durant les derniers mois de l'automne, ce qui porte à croire que l'exposition à la blastomycose durant le printemps et l'été est suivie par une longue période d'incubation.

Conclusion : Les régions du nord-ouest de l'Ontario signalent des taux élevés de blastomycose. On ignore dans quelle mesure il existe des différences régionales dans d'autres États et provinces. Les différences interrégionales peuvent justifier la hiérarchisation des stratégies de prévention et de contrôle de la blastomycose ainsi que l'intensification de la recherche et de la surveillance.

\section{Affiliations}

${ }^{1}$ Anciennement de la Northwestern Health Unit de Kenora (Ontario)

${ }^{2}$ Northwestern Health Unit de Kenora (Ontario)

*Correspondance : stefan. litvinjenko@mail.utoronto.ca

Citation proposée : Litvinjenko S, Lunny D. Hospitalisations pour une blastomycose dans le nord-ouest de l'Ontario, de 2006 à 2015. Relevé des maladies transmissibles au Canada. 2017;43(10):226-31.

https://doi.org/10.14745/ccdr.v43i10a02f

\section{Introduction}

La blasmocytose, causée par l'organisme Blastomyces dermatitidis, est une maladie fongique invasive dont le seul réservoir naturel connu se trouve dans le sol. Les cas de blastomycose se produisent principalement dans les régions est de l'Amérique du Nord, dans les provinces et les États qui longent les Grands Lacs (c.-à-d. Ontario, Michigan, Wisconsin, Minnesota), mais certains ont aussi été observés dans le Midwest des États-Unis et le centre du Canada (1). II est rare que des cas soient signalés à l'extérieur de l'Amérique du Nord.

La maladie serait symptomatique dans environ $50 \%$ des cas (2), ce qui laisse croire que les personnes en bonne santé sont assez résistantes à la progression néfaste du champignon invasif. On estime que $70 \%$ des cas peuvent être attribués à la blastomycose pulmonaire, qui se manifeste habituellement comme une grippe; les symptômes peuvent souvent entraîner un diagnostic erroné d'autres maladies, par exemple la tuberculose. La maladie extrapulmonaire se manifeste le plus souvent sous forme de blastomycose cutanée, mais elle peut aussi toucher l'appareil génito-urinaire ainsi que le système squelettique et le système nerveux central. Grâce à un traitement antifongique ou chirurgical approprié, le taux de mortalité de la blastomycose varie de 5 à $10 \%$ (2).

La période d'incubation moyenne de la blastomycose est généralement reconnue pour durer de 30 à 45 jours, mais cet intervalle peut être de 13 à 106 jours, selon la taille de l'inoculum (3) et la forme de la maladie (4). Bien que la 
blastomycose se contracte principalement par l'inhalation de spores en suspension dans l'air, elle peut aussi se contracter, quoique rarement, par une matière infectieuse en contact avec une perforation de la peau. L'exposition aux spores fongiques peut augmenter lors d'activités d'excavation et de construction ainsi que lors d'activités récréatives qui comportent un contact avec le sol près de plans d'eau, car l'environnement humide et acide du sol est favorable à la croissance de $B$. dermatitidis $(2,5)$. L'aérosolisation des spores se produit plus facilement lorsque le sol est remué à l'état sec (2). En raison des conditions climatiques variables (c.-à-d. précipitations, température et humidité) et des effets sur la composition du sol (c.-à-d. pH et contenu organique), il peut être difficile $\mathrm{d}^{\prime}$ isoler $B$. dermatitidis de l'environnement $(2,3,6)$.

En Ontario, la blastomycose n'est plus une maladie à déclaration obligatoire depuis 1989 (en raison de la transmissibilité limitée et du faible nombre de cas signalés), mais elle est encore à déclaration obligatoire au Manitoba (7). La blastomycose est fréquente dans le centre et le Midwest des États-Unis (8) et demeure une maladie à déclaration obligatoire en Arkansas, en Louisiane, au Michigan, au Minnesota et au Wisconsin (9). Selon Santé Canada, le taux d'incidence annuel de la blastomycose en Ontario, au Québec, au Manitoba et en Saskatchewan est de 0,62 cas pour 100000 personnes, certaines régions enregistrant des taux d'hospitalisation de 0,3 à 0,6 cas pour 100000 personnes (10).

Une étude de 2005 sur la blastomycose dans le nord-ouest de I'Ontario a révélé un taux d'incidence annuel élevé de 17,0 cas pour 100000 personnes de 1989 à 2005 (Mann V, Limerick B, Wiebe L. Northwestern Health Unit blastomycosis cases, 1989 to 2005: preliminary analysis. 2005; données non publiées). Lors d'une étude menée sur une plus courte période (de 1997 à 1999) dont les résultats ont été publiés antérieurement, on avait calculé un taux annuel de blastomycose de 117,2 cas pour 100000 personnes au moment d'une éclosion à Kenora (11). Ce taux est considéré comme étant exceptionnellement élevé, tout comme le taux de 404,9 cas pour 100000 personnes vivant sur une réserve (11).

L'objectif de la présente étude est d'utiliser les données sur I'hospitalisation de la dernière décennie (de 2006 à 2015) afin de décrire les récentes tendances épidémiologiques de I'hospitalisation pour une blastomycose dans le nord-ouest de I'Ontario.

\section{Méthodologie}

Cette analyse se concentre sur les cas hospitalisés de blastomycose dans le nord-ouest de l'Ontario. Aux fins du présent rapport, le terme "nord-ouest de l'Ontario " se rapporte à la circonscription hospitalière de la Northwestern Health Unit (NWHU). La circonscription hospitalière de la NWHU est I'un des 36 bureaux de santé publique de l'Ontario. Elle sert une population de près de 82000 personnes sur une superficie de 171288 kilomètres carrés (à peu près le cinquième de la taille de la province). La région englobe une partie du district de Kenora et tout le district de Rainy River, et la présente analyse porte sur Kenora, Dryden, Red Lake, Sioux Lookout, Rainy River, Emo, Fort Frances et Atikokan. Ces régions comprennent les municipalités nommées ainsi que de plus petites collectivités voisines et des réserves de Premières nations. La circonscription hospitalière de la NWHU compte 39 Premières Nations reconnues, certaines étant situées autour des principales municipalités, alors que d'autres sont plus au nord et moins accessibles.

\section{Critères d'inclusion}

L'échantillon de l'étude comprenait les dossiers d'hospitalisation pour toute forme de blastomycose diagnostiquée chez des résidents de l'Ontario de 2006 à 2015, répertoriés à l'aide des codes de la CIM-10 de B40.0 à B40.9 comme diagnostic primaire (12).

Le lieu de I'hospitalisation correspondait au lieu de résidence des patients plutôt qu'à l'endroit où ils étaient hospitalisés. Par exemple, si un patient du nord-ouest de l'Ontario était hospitalisé à Toronto, l'hospitalisation était classée dans le nord-ouest de l'Ontario. Cependant, les hospitalisations hors de l'Ontario n'ont pas été comptabilisées dans les données et étaient inaccessibles.

\section{Sources des données}

Les données sur les hospitalisations pour une blastomycose ont été extraites le 20 janvier 2017 de la base de données sur les congés des patients (BDCP). La BDCP a été consultée par l'intermédiaire de Savoir-Santé Ontario, un recueil de connaissances exploité par le ministère de la Santé et des Soins de longue durée. La BDCP comprend des renseignements administratifs, cliniques et démographiques sur les congés fournis par l'Institut canadien d'information sur la santé (ICIS). Savoir-Santé Ontario est exploité par le ministère de la Santé et des Soins de longue durée.

Les estimations de la population de la circonscription hospitalière de la NWHU proviennent de Statistique Canada et ont été consultées par l'intermédiaire de Savoir-Santé Ontario.

\section{Nombres et taux d'hospitalisations}

Le nombre d'hospitalisations pour une blastomycose a été calculé pour chacun des 36 bureaux de santé publique de l'Ontario. Ces résultats ont permis de calculer les taux d'hospitalisation des résidents du nord-ouest de l'Ontario ainsi que de plus petites zones géographiques de cette région.

Les taux d'hospitalisation bruts et selon l'âge ont été calculés en divisant le nombre d'hospitalisations s'étant produites au cours de la période par le total d'années-personnes à risque, puis en multipliant le résultat par 100000 années-personnes. Un taux exprimé pour 100000 années-personnes indique le nombre d'hospitalisations ayant eu lieu dans une population de 100000 personnes au cours d'un an.

Les taux d'hospitalisation normalisés selon l'âge ont été calculés en fonction de la population du recensement canadien de 2011 comme population de référence. Toutes les analyses ont été effectuées à l'aide des logiciels Excel, Open Epi et Epilnfo7.

\section{Taux du nord-ouest de l'Ontario}

Les taux d'hospitalisation normalisés selon l'âge pour la population de la circonscription hospitalière de la NWHU et de 
ses sous-régions, ainsi que les intervalles de confiance (IC) à $95 \%$ correspondants, ont été calculés à l'aide de l'approximation de la distribution normale selon la loi de Poisson. Les rapports des taux ont été calculés et utilisés afin de repérer toute différence statistique des taux d'hospitalisation entre les sous-régions. Les résultats étaient considérés comme étant statistiquement significatifs si I'IC à $95 \%$ du rapport des taux ne contenait pas le nombre « un».

\section{Taux selon l'âge et le sexe}

Les taux d'hospitalisation selon l'âge et le sexe et les IC à $95 \%$ ont été calculés à l'aide de la méthode exacte mi-P. L'analyse a porté sur des groupes d'âge d'un intervalle de 10 ans (de 0 à 9 ans, de 10 à 19 ans, etc.). Les tests pour déterminer les différences statistiques des taux entre les strates d'âge et de sexe reposaient sur le calcul des rapports des taux. Les résultats étaient considérés comme étant statistiquement significatifs si I'IC à $95 \%$ du rapport des taux ne contenait pas le nombre " un ».

\section{Dénombrement par code diagnostique}

Le nombre d'hospitalisations catégorisées par type de blastomycose a été comptabilisé pour le nord-ouest de l'Ontario. Le dénombrement de chaque type de blastomycose était en fonction des codes diagnostiques de la CIM-10, qui allaient de B40.0 à B40.9 (12).

\section{Dénombrement par année et mois}

Le nombre d'hospitalisations a été examiné selon l'année et le mois de survenue. Les nombres totaux de mois cumulés sur la période de 10 ans de l'étude ont été calculés afin de déterminer le moment de l'année où les hospitalisations étaient les plus fréquentes.

\section{Résultats}

\section{Hospitalisations pour une blastomycose en Ontario}

De 2006 à 2015, un total de 581 hospitalisations pour une blastomycose ont été enregistrées par 29 des 36 bureaux de santé publique de l'Ontario (les sept autres bureaux n'ayant signalé aucun cas). Fait notable, 245 hospitalisations pour une blastomycose (42\%) ont été attribuées à des résidents du nord-ouest de l'Ontario seulement (c.-à-d. dans la NWHU) (figure 1).

\section{Hospitalisations dans le nord-ouest de l'Ontario selon la région}

Dans le nord-ouest de l'Ontario, de 2006 à 2015, le taux d'hospitalisation pour une blastomycose était de 35,0 pour 100000 personnes par année. Kenora affichait le plus haut taux d'hospitalisation à 57,9 pour 100000 personnes par année, et un rapport de taux statistiquement significatif de 3,13 (IC à $95 \%$ de 2,42 à 4,09; $p<0,01)$, comparativement au reste du nord-ouest de l'Ontario. En raison de leurs petits nombres, les hospitalisations dans les sous-régions d'Atikokan, d'Emo et de Red Lake ont été supprimées et ne sont pas prises en compte.
Figure 1 : Nombre d'hospitalisations pour une blastomycose par bureau de santé publique de l'Ontario, de 2006 à $2015(n=581)$

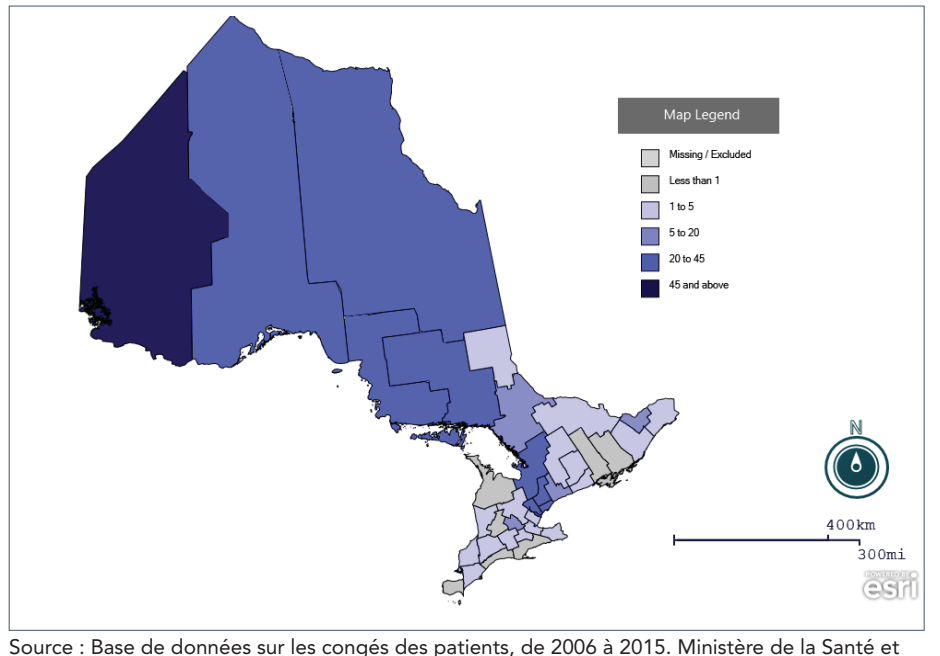
Soins de longue durée de l'Ontario. Savoir-Santé Or 2017. Remarque : L'image est disponible en anglais seulement

D'autres zones de la région affichaient des taux d'hospitalisation qui variaient de 16 à 32 pour 100000 personnes par année (figure 2).

Figure 2 : Nombre d'hospitalisations ${ }^{\star}$ pour une blastomycose selon la région du nord-ouest de l'Ontario', de 2006 à 2015

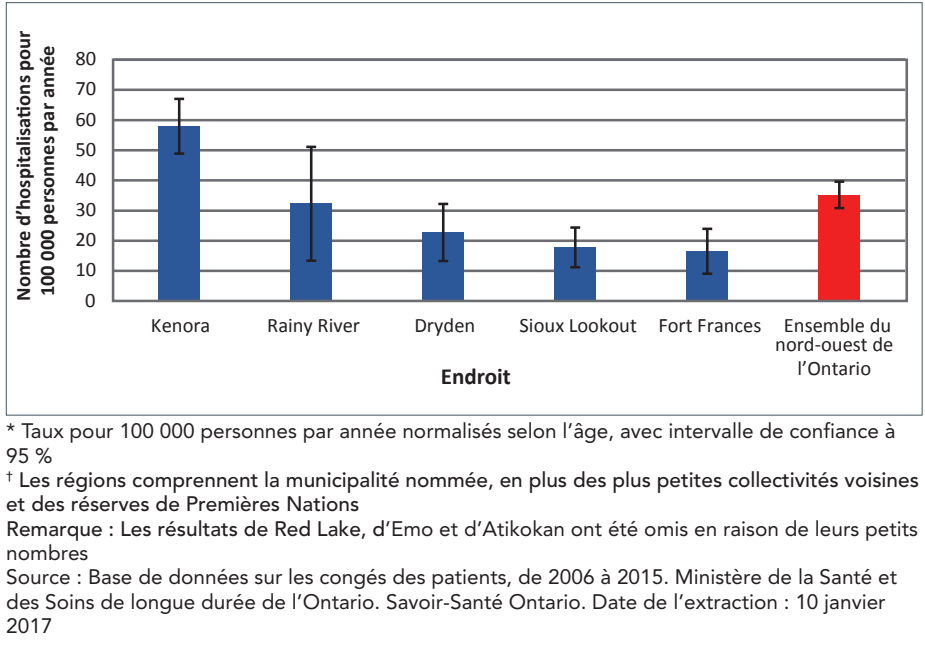

\section{Hospitalisations dans le nord-ouest de l'Ontario selon l'âge et le sexe}

Les hommes, qui représentaient $50,3 \%$ de la population de la circonscription hospitalière de la NWHU de 2006 à 2015, comptaient pour $58 \%(n=142)$ de l'ensemble des hospitalisations pour une blastomycose (comparativement à $n=103$ pour les femmes). Le taux d'hospitalisation en raison d'une blastomycose pour les hommes de tous âges était de 34,4 pour 100000 personnes par année, comparativement à 25,3 pour 100000 personnes par année pour les femmes. Cela 
équivaut à un rapport de taux statistiquement significatif de 1,36 (IC à $95 \%$ de 1,06 à 1,76; $p<0,05)$.

Le nombre d'hospitalisations pour une blastomycose était le plus faible chez les enfants de moins de 10 ans et les adultes âgés de 60 ans ou plus. Les taux étaient généralement les plus élevés chez les adultes dans la vingtaine jusqu'à la cinquantaine (figure 3). Les personnes dans la catégorie d'âge des 30 à 39 ans affichaient un rapport de taux statistiquement significatif de 2,04 (IC à $95 \%$ de 1,49 à 2,76; $p<0,01$ ), comparativement aux autres catégories d'âge. Toutefois, un examen des données a révélé que la surreprésentation des hommes avait gonflé cette estimation totale; le rapport des taux parmi les hommes de 30 à 39 ans était de 2,51 (IC à $95 \%$ de 1,70 à 3,66; $p<0,01$ ), comparativement aux femmes de ce groupe d'âge, chez qui les taux étaient insignifiants.

Figure 3 : Taux d'hospitalisation ${ }^{\star}$ pour une blastomycose selon l'âge et le sexe dans le nord-ouest de l'Ontario, de 2006 à 2015

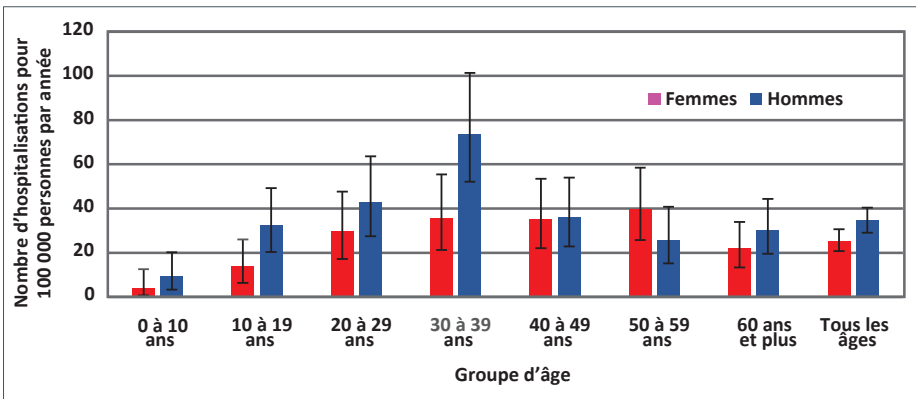

*Taux pour 100000 personnes par année, avec intervalle de confiance à $95 \%$

Source : Base de données sur les congés des patients, de 2006 à 2015. Ministère de la Santé et des Soins de longue durée. Savoir-Santé Ontario. Date de l'extraction : 10 janvier 2017

\section{Hospitalisations dans le nord-ouest de l'Ontario selon le code diagnostique de la CIM-10}

La majorité des hospitalisations pour blastomycose enregistrées de 2006 à 2015 (75\% du total des cas) était attribuable à la maladie pulmonaire (figure 4). Alors que la nature de certains cas ( 10\%) n'est pas précisée, on peut présumer que la plupart d'entre eux seraient également attribuables à une infection pulmonaire aiguë indiquant une infection temporaire. Cette proportion diffère de la maladie infectieuse chronique récidivante, qui compte pour $2,5 \%$ des hospitalisations connues. Les autres manifestations de la blastomycose, notamment les formes cutanée et disséminée, comptaient pour moins de $5 \%$ des hospitalisations connues.

Figure 4 : Hospitalisations pour une blastomycose selon le code diagnostique de la CIM-10 dans le nord-ouest de l'Ontario, de 2006 à 2015

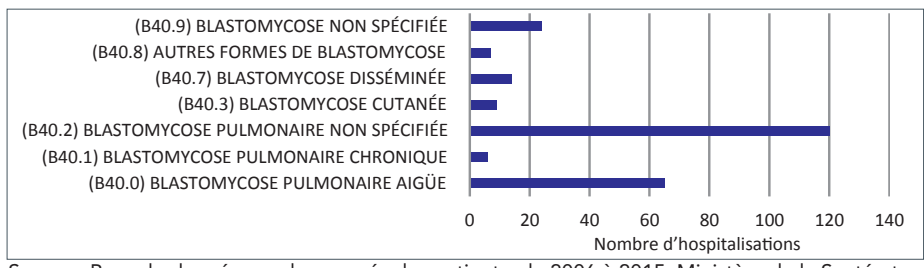

Source : Base de données sur les congés des patients, de 2006 à 2015. Ministère de la Santé et des Soins de longue durée de l'Ontario. Savoir-Santé Ontario. Date de l'extraction : 10 janvier 2017

\section{Hospitalisations dans le nord-ouest de l'Ontario selon l'année et le mois}

De 2006 à 2015, on a observé une légère augmentation du nombre d'hospitalisations pour une blastomycose dans le nord-ouest de l'Ontario (figure 5). Mis à part la tendance générale de la hausse du nombre d'hospitalisations, les années 2009 et 2013 ont enregistré des sommets, avec 41 hospitalisations dans les deux cas.

Figure 5 : Hospitalisations pour une blastomycose dans le nord-ouest de l'Ontario selon l'année, de 2006 à 2015

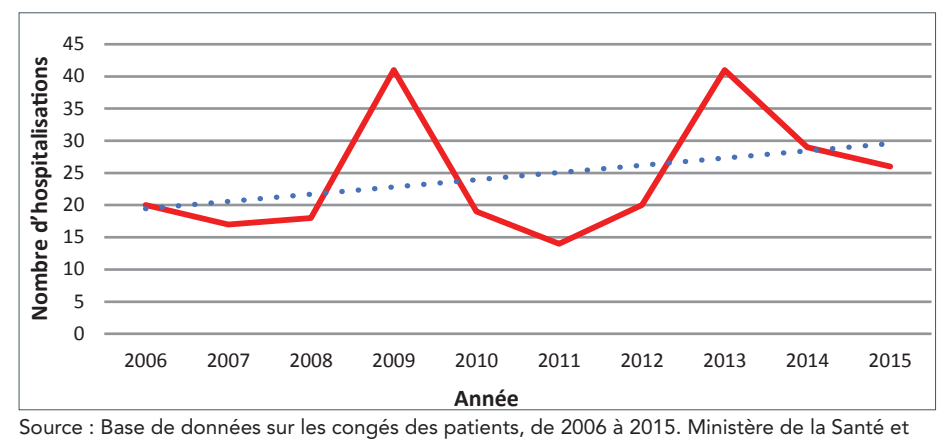

Source: Base de données sur les congés des patients, de 2006 à 2015. Ministère de la Santé et
des Soins de longue durée de l'Ontario. SavoirSanté Ontario. Date de l'extraction : 10 janvier 2017

De 2006 à 2015, il y a eu en moyenne plus d'hospitalisations pendant les mois de la fin de l'automne et du début de l'hiver que durant toute autre période de l'année (figure 6).

Figure 6 : Hospitalisations pour une blastomycose dans le nord-ouest de l'Ontario selon le mois, de 2006 à $2015(n=245)$

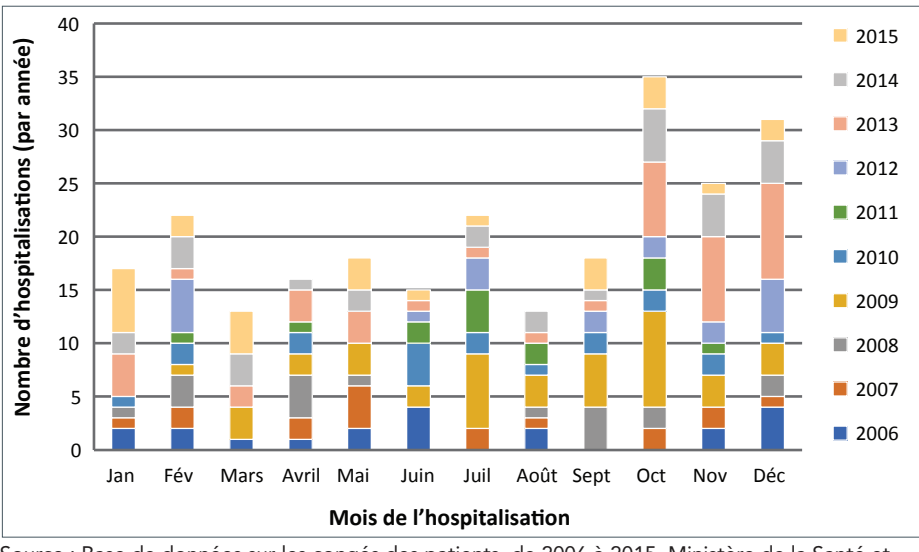

Source : Base de données sur les congés des patients, de 2006 à 2015. Ministère de la Santé et des Soins de longue durée de l'Ontario. Savoir-Santé Ontario. Date de l'extraction : 10 janvier 2017

\section{Discussion}

De 2006 à 2015, près de 600 hospitalisations pour une blastomycose ont été signalées en Ontario. Selon les estimations de la population de 2015, le nord-ouest de l'Ontario ne représente que $0,6 \%$ de la population de la province, mais compte pour $40 \%$ du nombre total d'hospitalisations durant la période de l'étude. Ces taux sont probablement une 
sous-estimation de la réelle incidence de la blastomycose, car la documentation porte à croire que seulement $50 \%$ des personnes atteintes de blastomycose présentent des symptômes cliniques menant à une hospitalisation (13). II semble exister une variation régionale importante au sein même du nord-ouest de l'Ontario, certaines régions sanitaires n'ayant signalé aucun cas au cours de cette période de 10 ans, alors que la région de Kenora affichait constamment un des taux d'hospitalisation pour une blastomycose les plus élevés de la province, faisant hausser les estimations régionales et provinciales de la morbidité. Combinés aux estimations antérieurement recensées dans la documentation, les taux élevés de la région de Kenora sont peut-être attribuables à des différences géologiques, comparativement aux régions est et sud du nord-ouest de I'Ontario, qui peuvent fournir des conditions plus favorables dans le sol pour la propagation de la blastomycose; toutefois, les types des sols n'ont pas été analysés dans le cadre de la présente étude. On ignore si de telles variations régionales sont aussi caractéristiques d'autres provinces canadiennes ou d'États américains.

En outre, le taux d'hospitalisation observé de 35,0 pour 100000 personnes par année dans le nord-ouest de l'Ontario demeure bien supérieur au taux annuel moyen le plus élevé signalé dans tout État américain, mais chevauche certaines données régionales aux États-Unis. Par exemple, bien qu'on ait signalé un taux ajusté selon l'âge de 2,9 hospitalisations pour 100000 personnes pour l'État du Wisconsin (16), certains comtés de cet État ont signalé de 10 à 40 cas pour 100000 personnes (17). II faut souligner que les augmentations moyennes annuelles des taux de blastomycose dans le nord-ouest de l'Ontario peuvent indiquer une réelle croissance de l'incidence de la maladie ou être le résultat d'une sensibilisation clinique et de méthodes d'examen améliorées de la part des médecins (12).

Le fait que la majorité des cas de blastomycose observés en Ontario de 2006 à 2015 était composée d'hommes (58\%) corrobore les données d'autres études. Cette observation peut être attribuée au fait que les hommes sont en nombre dominant dans les emplois des domaines de l'excavation et de la construction. Il est aussi possible que plus d'hommes que de femmes préfèrent les activités récréatives qui se pratiquent près des plans d'eau qui favorisent la croissance de $B$. dermatitidis (5). L'exception se trouvait dans le groupe d'âge des 50 à 59 ans, où $59 \%$ des cas étaient des femmes et $41 \%$, des hommes.

La plupart des hospitalisations pour une blastomycose étaient dans le groupe d'âge des 30 à 39 ans. Cette situation contraste avec celle observée au Manitoba, où les personnes âgées de 50 à 69 ans se partageaient le taux d'incidence le plus élevé (2). De même, un plus ancien rapport du nord-ouest de l'Ontario a révélé que les taux d'hospitalisation les plus élevés se retrouvaient chez les personnes du groupe d'âge des 40 à 59 ans (Mann $V$ et al., données non publiées). Sans égard à l'âge et au sexe, les tendances saisonnières correspondent à une exposition au cours du printemps ou de l'été et à une période d'incubation menant au diagnostic à la fin de l'automne.

\section{Forces et faiblesses}

Une des forces de cette étude était la qualité des données provenant de I'Institut canadien d'information sur la santé (ICIS), qui permettaient de détecter les différences de la maladie selon les régions et de fournir une population échantillon complète selon les hospitalisations (une vaste proportion de la documentation sur la blastomycose est fondée sur le signalement selon les éclosions). En ce qui concerne la validité, Santé publique Ontario a fourni à la NWHU des données sur les nombres de cultures positives confirmées en laboratoire de B. dermatitidis pour les années 2010 à 2015 qui, en théorie, seraient une mesure plus représentative de l'incidence de la blastomycose dans la région. Toutefois, comme les données sur les hospitalisations ont produit des chiffres comparables aux données sur les nombres des cultures confirmées en laboratoire, les données sur les hospitalisations ont été considérées comme mesure de substitution des hospitalisations associées à la blastomycose dans le nord-ouest de l'Ontario.

L'étude était limitée par l'utilisation des dossiers d'hospitalisation pour évaluer la blastomycose, qui ne pouvait être qu'un substitut à la réelle incidence, étant donné qu'on ne peut pas s'attendre à ce que tous cherchent à recevoir des soins ou y aient accès, ou encore que tous soient hospitalisés s'ils viennent chercher des soins médicaux. En outre, certains cas, en particulier les cas graves, sont envoyés à Winnipeg, au Manitoba; par conséquent, ils ne sont pas enregistrés en tant que cas en Ontario et peuvent brouiller les taux d'incidence au Manitoba entre les expositions attribuées à la région et les visites de l'extérieur de la province (14). De plus, alors que d'autres études ont révélé des taux de blastomycose significativement plus élevés dans les populations autochtones $(11,14,15)$, l'information sur le statut d'Autochtone des cas n'était pas accessible dans la population à l'étude. Des études futures permettant d'évaluer les données des dossiers individuels, de surveiller les facteurs de risques pertinents (p. ex. facteurs sociodémographiques), d'isoler les sources probables de l'infection et de déterminer les taux globaux d'incidence contribueraient à repousser les limites de la présente étude et d'accroître la compréhension de cette maladie.

\section{Conclusion}

Les régions du nord-ouest de l'Ontario signalent des taux élevés de blastomycose. On ignore dans quelle mesure il existe des différences régionales dans d'autres États et provinces. Les différences interrégionales peuvent justifier la hiérarchisation des stratégies de surveillance, de prévention et de contrôle de la blastomycose ainsi que l'intensification de la recherche.

\section{Déclaration des auteurs}

S.L. - Conceptualisation, méthodologie, analyse formelle, enquête, visualisation, rédaction - ébauche originale - examen et révision, administration du projet 
D.L. - Conceptualisation, méthodologie, validation, ressources, collecte de données, rédaction - examen et révision, supervision, administration du projet

\section{Conflit d'intérêt}

Aucun.

\section{Remerciements}

Nous aimerions remercier la $\mathrm{D}^{\text {re }}$ Kit Young Hoon, médecin hygiéniste de la Northwestern Health Unit, pour sa supervision, son expertise et sa validation du présent rapport. Merci aussi à tous ceux sur le terrain qui ont aidé à collecter les données et qui ont fourni des soins aux personnes atteintes de maladies infectieuses.

\section{Financement}

Cette recherche a été appuyée par la Northwestern Health Unit.

\section{Références}

1. Centers for Disease Control \& Prevention. Blastomycosis. Atlanta (GA): CDC. https://www.cdc.gov/fungal/diseases/ blastomycosis/ [Consulté le 8 fév 2017].

2. Manitoba Health Communicable Disease Control Unit. Communicable Disease Management Protocol: Blastomycosis. 2015. https://www.gov.mb.ca/health/ publichealth/cdc/protocol/blastomycosis.pdf

3. Klein BS, Vergeront JM, Weeks RJ, Kumar UN, Mathai G, Varkey B, Kaufman L, Bradsher RW, Stoebig JF, Davis JP. Isolation of Blastomyces dermatitidis in soil associated with a large outbreak of blastomycosis in Wisconsin. N Eng J Med. 1986;314:529-34. DOl (http://dx.doi.org/10.1056/ NEJM198602273140901). PubMed (https://www.ncbi.nlm. nih.gov/entrez/query.fcgi?cmd=Retrieve \&db=PubMed\&li st_uids $=3945290 \& d o p t=A b s t r a c t)$.

4. Gray NA, Baddour LM. Cutaneous inoculation blastomycosis. Clin Infect Dis. 2002;34(10):E44-9. DOI (http://dx.doi. org/10.1086/339957). PubMed (https://www.ncbi.nlm.nih. gov/entrez/query.fcgi?cmd=Retrieve\&db=PubMed\&lis t_uids $=11981746 \& d o p t=$ Abstract $)$.

5. Klein BS, Vergeront JM, DiSalvo AF, Kaufman L, Davis JP. Two outbreaks of blastomycosis along rivers in Wisconsin: isolation of Blastomyces dermatitidis from riverbank soil and evidence of its transmission along waterways Am Rev Respir Dis. 1987;136(6):1333-8. DOI (http://dx.doi.org/10.1164/ ajrccm/136.6.1333). PubMed (https://www.ncbi.nlm.nih gov/entrez/query.fcgi?cmd=Retrieve\&db=PubMed\&lis t_uids=3688635\&dopt=Abstract).

6. Morris SK, Brophy J, Richardson SE, Summerbell R, Parkin PC, Jamieson F, Limerick B, Wiebe L, Ford-Jones EL. Blastomycosis in Ontario, 1994-2003. Emerg Infect Dis.,2006;12(2):274-9. DOI (http://dx.doi.org/10.3201/ eid1202.050849). PubMed (https://www.ncbi.nlm.nih. gov/entrez/query.fcgi?cmd=Retrieve\&db=PubMed\&lis t_uids=16494754\&dopt=Abstract).
7. Population and Public Health Branch Summary of reportable diseases 1990. Toronto: Communicable Disease Control, Ontario Ministry of Health; 1991.

8. Seitz AE, Adjemian J, Steiner CA, Prevots DR Spatial epidemiology of blastomycosis hospitalizations: detecting clusters and identifying environmental risk factors. Med Mycol. 2015 Jun;53(5):447-54. DOI (http://dx.doi. org/10.1093/mmy/myv014). PubMed (https://www.ncbi.nlm. nih.gov/entrez/query.fcgi?cmd=Retrieve\&db=PubMed\&li st_uids $=25908653 \&$ dopt $=$ Abstract).

9. Centers for Disease Control \& Prevention. Reportable fungal diseases by state. Atlanta (GA): CDC. https://www.cdc.gov/ fungal/fungal-disease-reporting-table.html [Consulté le 8 fév 2017].

10. Gouvernement du Canada. Surveillance de la blastomycose. Ottawa (ON): Santé Canada. https://www.canada.ca/fr/ sante-publique/services/maladies/blastomycose/surveillanceblastomycose.html [Consulté le 8 fév 2017].

11. Dwight PJ, Naus M, Sarsfield P, Limerick B. Éclosion de blastomycose humaine : épidémiologie de la blastomycose dans le bassin démographique de Kenora en Ontario, Canada. Relevé des maladies transmissibles. 2000;26(10):82-91. PubMed (https://www.ncbi.nlm.nih. gov/entrez/query.fcgi?cmd=Retrieve\& $\mathrm{db}=$ PubMed\&lis t_uids=10893821\&dopt=Abstract).

12. World Health Organization. International statistical classification of diseases and health related problems, 10th revision. Geneva: World Health Organization. 2016.

13. Chapman SW, Dismukes WE, Proia LA, Bradsher RW, Pappas PG, Threlkeld MG, Kauffman CA; Infectious Diseases Society of America. Clinical practice guidelines for the management of blastomycosis: 2008 update by the Infectious Diseases Society of America. Clin Infect Dis. 2008;46(12):1801-12. DOI (http://dx.doi.org/10.1086/588300). PubMed (https:// www.ncbi.nlm.nih.gov/entrez/query.fcgi?cmd=Retrieve\&db= PubMed\&list_uids=18462107\&dopt=Abstract).

14. Crampton TL, Light RB, Berg GM, Meyers MP, Schroeder GC, Hershfield ES, Embil JM. Epidemiology and clinical spectrum of blastomycosis diagnosed at Manitoba hospitals. Clin Infect Dis. 2002;34(10):1310-6. DOI (http://dx.doi. org/10.1086/340049). PubMed (https://www.ncbi.nlm.nih. gov/entrez/query.fcgi?cmd=Retrieve $\& \mathrm{db}=$ PubMed\&lis t_uids=11981725\&dopt=Abstract).

15. Dalcin D, Ahmed S. Blastomycosis in northwestern Ontario, 2004 to 2014. Can J Infect Dis Med Microbiol. 2015;26(5):259-62. DOI (http://dx.doi. org/10.1155/2015/468453). PubMed (https://www.ncbi.nlm. nih.gov/entrez/query.fcgi?cmd=Retrieve \&db=PubMed\&li st_uids=26600814\&dopt=Abstract).

16. Seitz A, Younes N, Steiner C, Prevots D. Incidence and trends of blastomycosis-associated hospitalizations in the United States. PLoS One. 2014;9(8):e105466. DOI (http://dx.doi. org/10.1371/journal.pone.0105466). PubMed (https://www. ncbi.nlm.nih.gov/entrez/query.fcgi?cmd=Retrieve\&db=PubM ed\&list_uids=25126839\&dopt=Abstract).

17. Benedict K, Roy M, Chiller T, Davis J. Epidemiologic and Ecologic features of blastomycosis: a review. Curr Fungal Infect Rep. 2012;6(4):327-35. DOI (http://dx.doi.org/10.1007/ s12281-012-0110-1). 